\title{
Intraluminal Route of Administration
}

National Cancer Institute

\section{Source}

National Cancer Institute. Intraluminal Route of Administration. NCI Thesaurus. Code C38251.

Administration of a drug within the cavity or channel of a tubular structure or tubular organ. 